\title{
Comparative Analysis of Circular and Triangular Gold Nanodisks for Field Enhancement Applications
}

\author{
Karlo Q. da Costa, Victor Dmitriev, \\ Federal University of Para,Belém-PA, Brazil,e-mails: karlo@ufpa.br, victor@ufpa.br
}

\begin{abstract}
In this paper, we present a quantitative comparison of circular and triangular gold nanodisks with the same length and thickness. The method of moments is used to solve numerically the scattering problem. With this model, we investigate the spatial near field distribution, spectral response, far field diagrams, and bandwidth wavelength of these particles. Our results show that the resonant wavelength and the near field enhancement and confinement of the triangular particle are larger than those for the circular particle, but the resonance bandwidth and scattering cross section of the triangular particle are smaller.
\end{abstract}

Index Terms - Plasmonics, metal nanoparticles, optical scattering, near and far field analysis.

\section{INTRODUCTION}

The electromagnetic scattering of metals in optical frequency region possesses special characteristics. At these frequencies, there are electron oscillations in the metal called plasmons with distinct resonant frequencies, which produce strongly enhanced near fields at the metal surface. This effect can be analyzed using Lorentz-Drude model of the complex dielectric constant. The science of the electromagnetic optical response of metal nanostructures is known as plasmonics or nanoplasmonics [1].

One of the possible applications of plasmonics is design of nanoantennas [2]-[7] which are metal nanostructures used to confine and enhance optical electromagnetic fields. An optical monopole antenna is investigated in [3]. In [4], Bowtie optical antennas are analyzed. Dipoles nanoantennas are presented in [5], and sphere nanoantennas are discussed in [6], [7]. Examples of applications of these antennas are ultra-high-density data storage, super-resolution microscopy, integrated nano-optical devices and surface-enhanced Raman scattering [1]. Most of these antennas are composed of coupled metal nanoparticles. To understand the electromagnetic behavior of these nanoantennas it is important to investigate the resonances and field distributions of individual particles. Some common metal nanoparticles have been analyzed, e.g. spheres [7], circular disk [8], and triangular disk [9], but to the best of our knowledge there is no precise comparative study of these particles in terms of near and far field characteristics.

In this paper, we present a quantitative comparison of two nanoparticles with different shapes in terms of resonant properties, near and far field distributions, and wavelength bandwidth. The particles 
considered in this paper are circular and triangular disks with equal length and thickness. There are some computational methods in the literature used to analyze these particles, e.g. the discrete dipole approximation (DDA), the finite difference time domain method (FDTD) [10], and the boundary element method (BEM). We apply the method of moments (MoM) to calculate the optical scattering of these particles [11]. The Lorentz-Drude theory with one interband term is used to model the complex permittivity of the gold. To test the MoM algorithm, we compare our numerical results with the analytical Mie theory for the gold metal sphere.

\section{THEORY}

The gold nanoparticles analyzed in this paper are shown in Fig. 1, where two planar geometries are presented, namely triangular and circular disks. The length and thickness of these particles are $L$ and $H$, respectively. These particles are illuminated by an Ex-polarized, $z$-directed plane wave and the surrounded medium is free space.

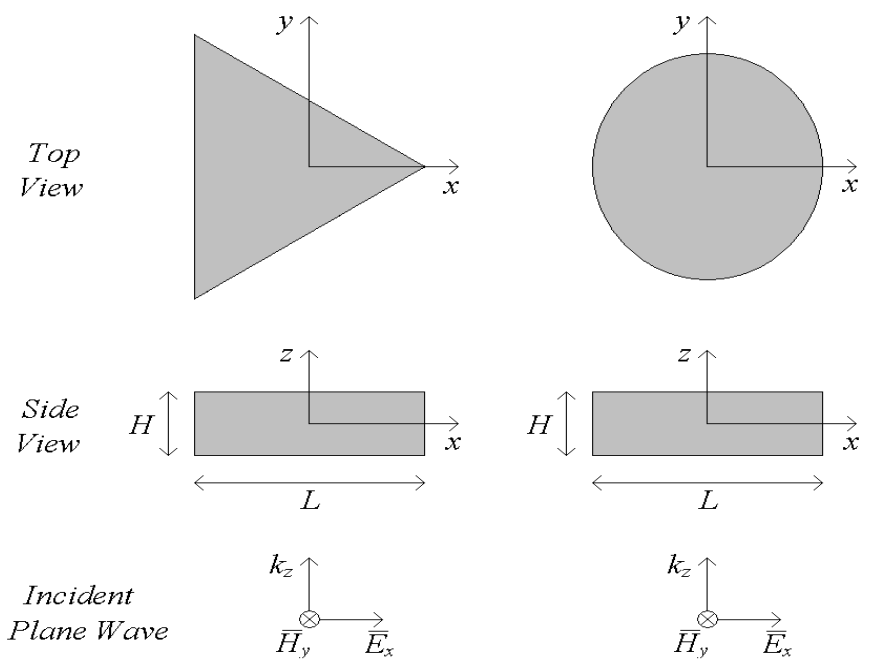

Fig. 1. Geometries of the analyzed gold triangular disk (left) and circular disk (right).

The numerical analysis of the scattering problems shown in Fig. 1 was fulfilled by our MoM code based on the model proposed in [11], where the equivalent polarization current inside the volume of the particles

$$
\bar{J}_{e q}=j \omega\left[\varepsilon(\bar{r})-\varepsilon_{0}\right] \bar{E}(\bar{r})=\tau(\bar{r}) \bar{E}(\bar{r})
$$

is determined by solving the tensor integral equation for the electric field

$$
\left[1+\frac{\tau(\bar{r})}{3 j \omega \varepsilon_{0}}\right] \bar{E}(\bar{r})-P V \int_{v} \tau(\bar{r},) \bar{E}(\bar{r}) \cdot \bar{G}\left(\bar{r}, \bar{r}^{\prime}\right) d v^{\prime}=\bar{E}^{i}(\bar{r})
$$

In (2), $P V$ means the principal value of the integral where the evaluation is inside the volume $v$ 
excluding the singularities of the free-space vector Green's function $\bar{G}$. The variables and parameters in (1)-(2) are as follows: $\bar{E}^{i}$ and $\bar{E}$ are the incident and total electric field inside the volume $v$, respectively, $\omega$ the angular frequency, $k_{0}=\omega\left(\mu_{0} \varepsilon_{0}\right)^{1 / 2}=2 \pi c / \lambda$ the wave number, $\lambda$ is the wavelength, $c$ is the speed of the light in free space, $\mu_{0}$ the free space permeability, $\varepsilon_{0}$ the free space permittivity. The complex permittivity of the Au particles is $\varepsilon=\varepsilon_{0} \varepsilon_{r}$, where $\varepsilon_{r}$ is defined

$$
\varepsilon_{r}=1-\frac{\omega_{p 1}^{2}}{\omega^{2}-j \Gamma \omega}+\frac{\omega_{p 2}^{2}}{\omega_{0}^{2}-\omega^{2}+j \gamma \omega}+\varepsilon_{\infty}
$$

and the parameters of this equation are: $\varepsilon_{\infty}=6, \omega_{p 1}=13.8 \times 1015 \mathrm{~s}^{-1}, \Gamma=1.075 \times 1014 \mathrm{~s}^{-1}, \omega_{0}=2 \pi c / \lambda_{0}$, $\lambda_{0}=450 \mathrm{~nm}, \omega_{p 2}=45 \times 1014 \mathrm{~s}^{-1}$, and $\gamma=9 \times 1014 \mathrm{~s}^{-1}$ [1]. This model is a good approximation in the range of wavelengths from $500 \mathrm{~nm}$ to $1000 \mathrm{~nm}$. Exactly in this frequency range we fulfill our analysis.

In this model, the volume of a particle is divided in $N$ small cubic subvolumes, where the total electric field is approximately constant. With this approximation, the integral equation is transformed into a linear system with $N_{t}=3 N$ equations because there are three electric field components in each subvolume. The solution of the equivalent linear system produces the total electric field $\bar{E}$ inside the volume $v$ of the particle. With this result, the near and far field characteristic parameters of the particles are calculated. We calculated the near electric field and scattering cross section respectively by

$$
\begin{gathered}
\bar{E}^{t}(\bar{r})=\bar{E}^{i}(\bar{r})+\int_{v} \tau\left(\bar{r}^{\prime}\right) \bar{E}\left(\bar{r}^{\prime}\right) \cdot \bar{G}\left(\bar{r}, \bar{r}^{\prime}\right) d v^{\prime} \\
S C S(\theta, \phi)=8 \pi \eta \frac{U(\theta, \phi)}{\left|\bar{E}^{i}\right|^{2}}
\end{gathered}
$$

where $\eta$ is the free space impedance and $U(\theta, \phi)$ is the radiation intensity of the scattered far field in a given direction $\theta$ and $\phi$. The total scattering cross section is defined by $S C S_{\text {total }}=2 \eta P_{s c} /\left|E_{i}\right|^{2}$, where $P_{s c}$ is the total power scattered by the particle [12].

The particles considered in this work are lossy and possess small $Q$ factor, this is why we prefer using the fractional wavelength bandwidth $(\Delta \lambda)$ to quantify the bandwidth around its resonances. We define this characteristic as $\Delta \lambda(\%)=200 \times\left(\lambda_{s}-\lambda_{i}\right) /\left(\lambda_{s}+\lambda_{i}\right)$, where $\lambda_{s}$ and $\lambda_{i}$ the superior and inferior wavelengths at $3 \mathrm{~dB}$ points, respectively, around the resonant wavelength where the normalized field intensity is reduced by a factor of $2^{-1 / 2}$ with respect to its maximum value at the resonance.

\section{NUMERICAL RESULTS}

\section{A. Analysis of one Gold Sphere}

For testing the convergence of the developed algorithm, we compare our numerical calculations with the exact Mie scattering theory for the case of the gold metal sphere [13]. In this analysis, one $\mathrm{Au}$ sphere with radius $a=60 \mathrm{~nm}$ for $\lambda=550 \mathrm{~nm}$ placed in a free space is positioned in the origin of the 
coordinate system and illuminated by Ex-polarized $z$-directed plane wave. Fig. 2 shows the results of the total electric field distributions near the sphere calculated by MoM with $N_{t}=11085$ and by using Mie series. The electric field is normalized with the amplitude of the electric field of the incident plane wave $\left|E_{x 0}\right|$. Only $x$ - components of the field are presented because the $y$ - and $z$-ones are very small. We observe in this figure a good concordance of the results at the points near and far from the sphere. However, there is a small difference between the results at the sphere's surface. This happens because there is a strong variation of the field near the sphere. Better agreement can be obtained with higher values of $N_{t}$ leading to the increased computational cost. But in practical applications, for example, in optical probes, any given particle or structure to be probed will be positioned at a certain distance from the surface of metal structures.
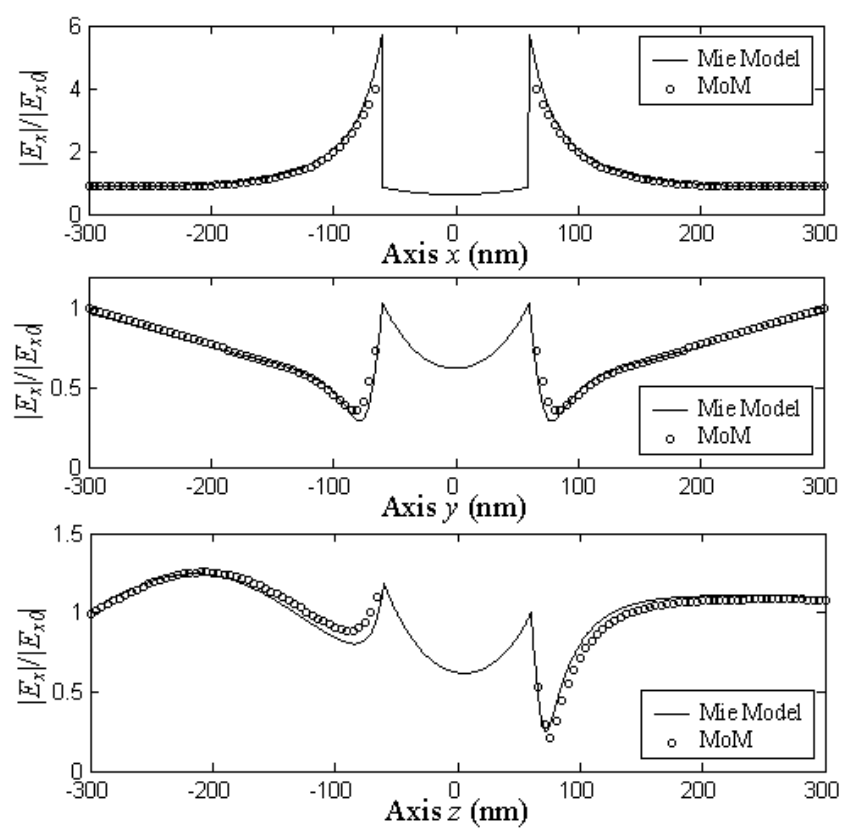

Fig. 2. Normalized near field distributions for $\lambda=550 \mathrm{~nm}$ along the axis $x$ (upper figure), $y$ (middle figure) and $z$ (lower figure) for gold sphere with radius $a=60 \mathrm{~nm}$.

\section{B. Resonant Response}

Fig. 3 shows the resonant responses of the nanoparticles presented in Fig. 1 and that of the sphere. In these simulations, the dimensions of the particles are $L=120 \mathrm{~nm}, H=20 \mathrm{~nm}$, and $2 a=120 \mathrm{~nm}$. This figure presents variation of the normalized electric field near the particles in function of the wavelength and distance $\mathrm{d}$ from the particles along the axis $x$. The point of calculation is $(L / 2+d, 0,0)$. 

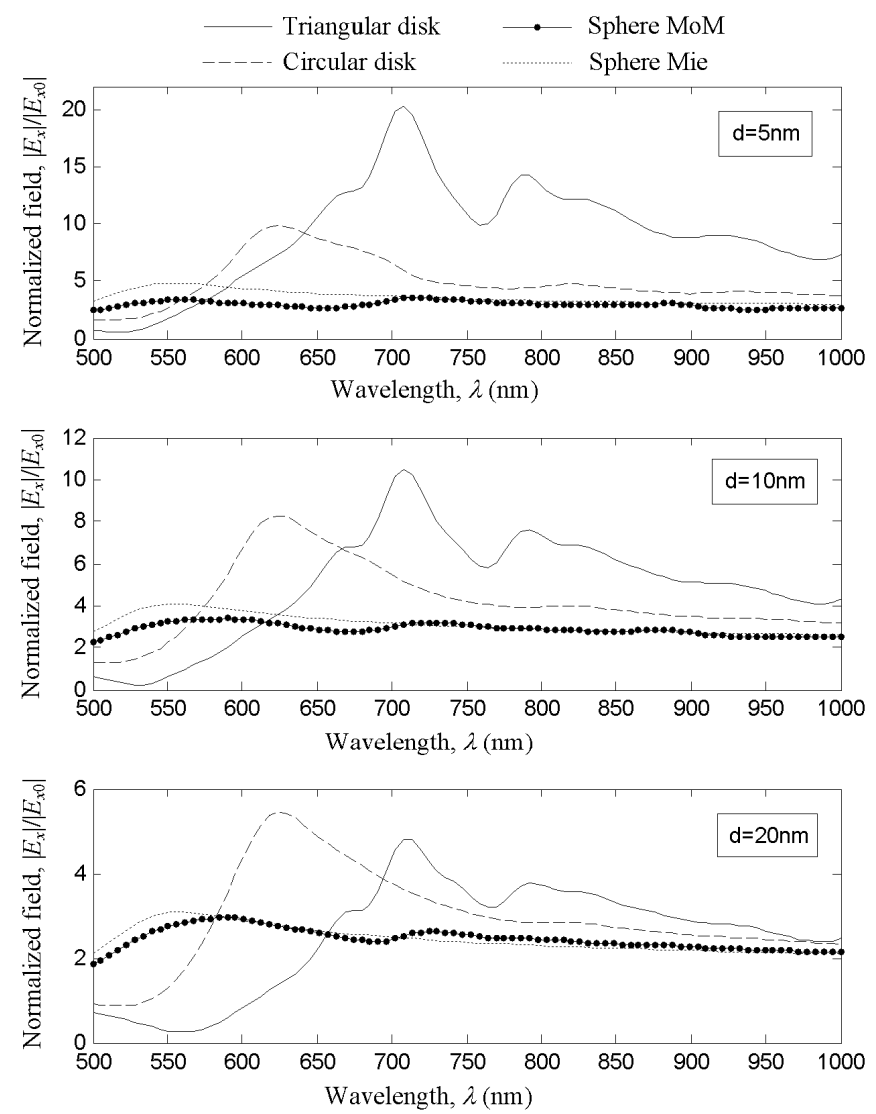

Fig. 3. Resonant response of the normalized field in different points along de axis $x(L / 2+\mathrm{d}, 0,0)$ for the sphere, circular, and triangular disks with $L=120 \mathrm{~nm}$.

Examining the results of this figure one can note a difference in resonances of the particles. For the triangular disk, the principal resonance is around the point $\lambda_{\text {res }}=707 \mathrm{~nm}$, for the circular disk is near $\lambda_{\text {res }}=623 \mathrm{~nm}$, and for the sphere is about $\lambda_{\text {res }}=555 \mathrm{~nm}$. These results show that the particles with accentuated tips have larger resonant wavelengths. In the spherical case, the analytical and numerical curves are a little different because of the error due discretization of the numerical method, but in this case there are one resonance. In the triangular disk case, the longitudinal dimension $L$ and the side of the triangle given by $2 L /(3)^{0.5}$ produce two principal resonances and some secondary ones that are common for this geometry [5], [14].

We can also observe from Fig. 3 that for the particles with more accentuated tips, e.g. triangular disk, the field enhancement is larger near the particles, which is a well known result. For example, at the distance $d=5 \mathrm{~nm}$ the maximum normalized electric field for the triangular particle is about two times larger $\left(\left|E_{x}\right| /\left|E_{x 0}\right| \approx 20.3\right)$ than that of the circular $\left(\left|E_{x}\right| /\left|E_{x 0}\right| \approx 9.8\right)$ one and four times larger than of spherical $\left(\left|E_{x}\right| /\left|E_{x 0}\right| \approx 4.8\right)$ one, but this field intensity decreases rapidly with the distance $d$. This means that the triangular disk possesses a smaller region near the surface's particle where the electric field is concentrated, i.e., this triangular disk has a more field confinement in comparison with the other two particles.

Another difference between these particles is the fractional wavelength bandwidth. For the case $d=5 \mathrm{~nm}$ shown in Fig. 3, the triangular particle possesses smaller bandwidth $(\Delta \lambda \approx 6.3 \%)$, around the 
principal resonance, in comparison with that for the circular particle $(\Delta \lambda \approx 15.7 \%)$.

Table I shows a comparison between characteristics of the particles: resonant wavelength, normalized near field enhancement $\left|E_{x}\right|^{2} /\left|E_{x 0}\right|^{2}$ at $d=5,10,20 \mathrm{~nm}$, scattering cross section SCS in $+z$ direction, total scattering cross section $S C S_{\text {total }}$, and wavelength bandwidth. All these characteristics were calculated for the principal near field resonant wavelength of the corresponding particle. The results presented in this table show that the triangular particle possess a larger maximum near field enhancement than the circular particle, but a smaller bandwidth and scattering cross sections.

TABLE I. SOME OPTICAL CHARACTERISTICS OF THE PARTICLES: SPHERE, CIRCULAR DISK, AND TRIANGULAR DISK

\begin{tabular}{llll}
\hline Characteristic & Sphere & Circular disk & Triangular disk \\
\hline Resonant wavelength $(\mathrm{nm})$ & 555 & 623 & 707 \\
Field enhancement at $d=5 \mathrm{~nm},\left|E_{x}\right|^{2} /\left|E_{x 0}\right|^{2}$ & 23 & 96 & 414 \\
Field enhancement at $d=10 \mathrm{~nm},\left|E_{x}\right|^{2} /\left|E_{x 0}\right|^{2}$ & 17 & 69 & 111 \\
Field enhancement at $d=20 \mathrm{~nm},\left|E_{x}\right|^{2} /\left|E_{x 0}\right|^{2}$ & 10 & 30 & 23 \\
$S C S\left(\mathrm{~nm}^{2}\right)$ & $3.95 \times 10^{4}$ & $7.19 \times 10^{4}$ & $1.87 \times 10^{4}$ \\
$S C S_{\text {total }}\left(\mathrm{nm}^{2}\right)$ & $2.67 \times 10^{4}$ & $4.29 \times 10^{4}$ & $1.22 \times 10^{4}$ \\
Wavelength bandwidth, $\Delta \lambda(\%)$ & - & 15.7 & 6.3 \\
\hline
\end{tabular}

\section{Near Field Distributions}

To analyze the field confinement properties of the planar particles in Fig. 1, a two dimensional near field distribution is presented in Fig. 4. The magnitudes of the fields are normalized with the incident plane wave, and this figure is plotted in the plane $x y$. The distributions were calculated at the resonant wavelength of the particles, namely, for the circular disk for $\lambda_{\text {res }}=623 \mathrm{~nm}$ and for the triangular disk for $\lambda_{\text {res }}=707 \mathrm{~nm}$. In Fig. 4 , the polarization of the incident plane wave is also shown.

From this figure, we can observe the better field confinement and enhancement in the plane $x y$ for the triangular disk in comparison with the circular disk.

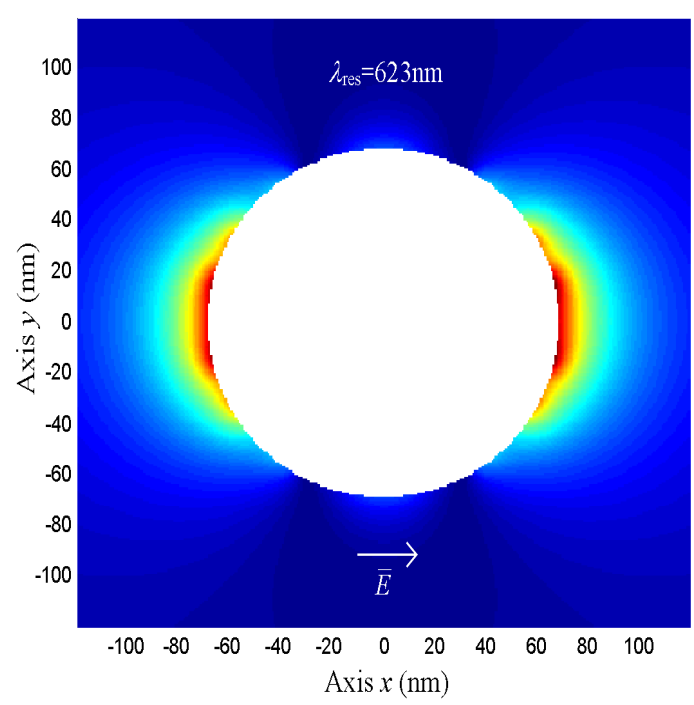

(a)
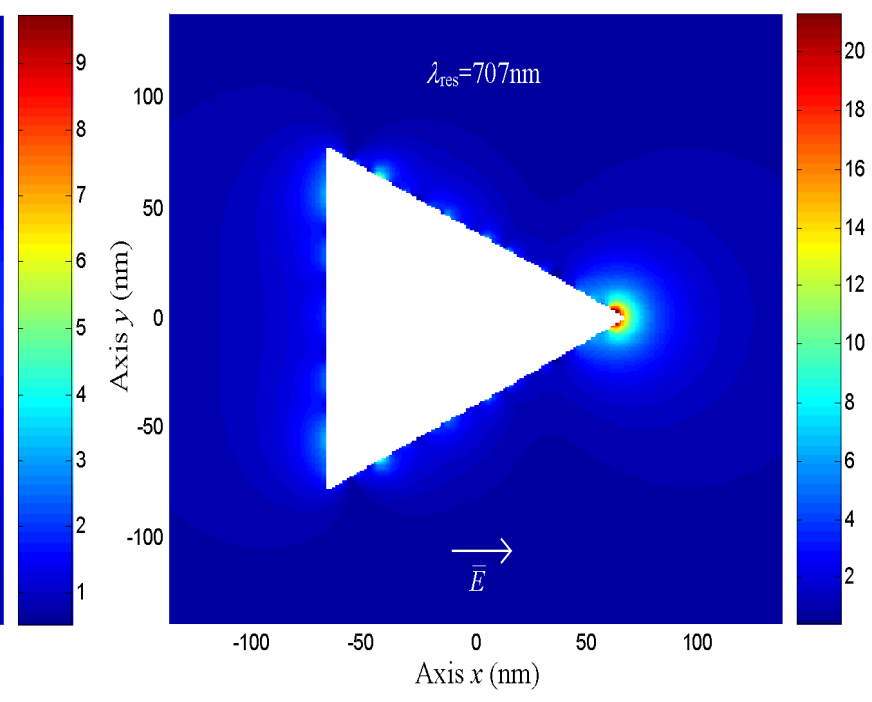

(b)

Fig. 4. Normalized field $\left(\left|E_{x}\right| /\left|E_{x 0}\right|\right)$ distributions of the (a) circular disk and (b) triangular disk at the plane $x y$. 


\section{Scattered Far Field}

Fig. 5(a) shows the variation of the SCS in the $+z$ direction (Fig. 1) versus wavelength for the circular and triangular disks. For comparison, the result for the spherical particle is also presented. Analyzing this figure, we observe that the resonance in far field for the three cases are similar to that in near field region (Fig. 3), but in general, the resonant behavior in the near and far field are different, because the forms of these fields are different [15]. In near field region, the evanescent field is predominant, and in the far field, only radiation field exists. Also, this figure shows that the SCS of the circular particle at the resonance is about four times larger than that for the triangular case.

Fig. 5(b) presents the normalized radiation diagrams of the particles. These diagrams are plotted in the plane $x z$ (Fig. 1), and were calculated for the resonant wavelengths presented in Table 1. This figure shows that all particles have the diagrams similar to those of small electric dipoles [12]. The circular and spherical particles have full rotational symmetry with respect to $z$, and the triangular particle has a lower symmetry, namely the three-fold one. This results in a little asymmetry in its radiation diagram.

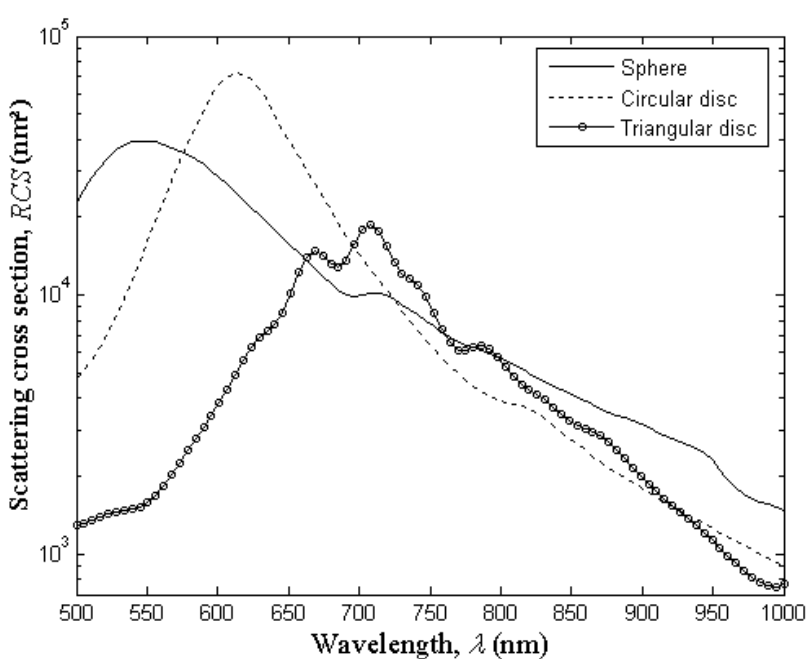

(a)

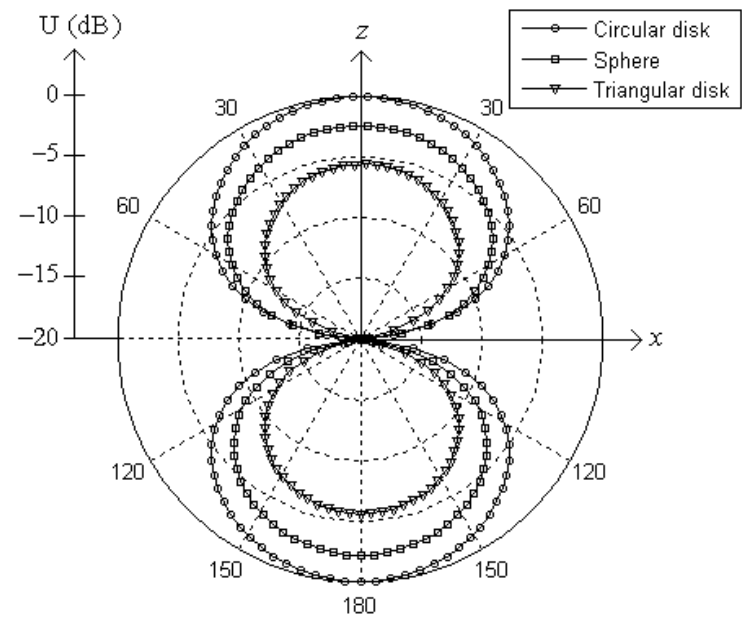

(b)

Fig. 5. (a) Scattering cross sections in the $+z$ direction versus wavelength. (b) Radiation intensity diagrams $U(\theta, \phi)$ of the particles in the plane $x z$ at respective resonant wavelength of Table 1. These diagrams are normalized with the maximum radiation intensity of the circular disk.

\section{CONCLUSIONS}

We presented in this paper a near and far field analysis for the circular and triangular gold nanodisks which have the same length and thickness. We observed that the triangular disk has a resonant wavelength larger than the circular one but the bandwidth of it is about two times smaller. We believe that these results are related with the volume and geometry of the particles, where particles with higher volume and smoother surface, i.e. without corner or tips, possess larger losses, weak resonance, and therefore broader bandwidth. This behavior is similar to that observed in microwaves antennas. On the other hand, the maximum near field enhancement of the triangular particle is about four times larger than that of the circular one and twenty times larger than of 
spherical one with the same length, but this field intensity decreases rapidly with the distance from the surface's particle. This result is due the electrostatic effect that exists in these kinds of problems, where particles with more accentuated tips possess higher electric fields near the tip's surface. In the far field region, we observed that the scattering cross section of the circular disk is about four times larger than that of the triangular disk. This happens because the area of the circular particle is higher than that of the triangular one.

\section{ACKNOWLEDGMENT}

This work was financially supported by the Brazilian agency National Counsel of Development Scientific and Technologic - CNPq by contract number 151731/2008-0.

\section{REFERENCES}

[1] L. Novotny, and B. Hecht, Principles of Nano-Optics, New York: Cambridge, 2006.

[2] D. W. Pohl, "Near field optics as an antenna problem," Near Field: Principles and Applications, The second AsiaPacific Workshop on Near Field Optics, Beijing, China, pp. 9-21, Oct. 1999.

[3] T. H. Taminiau, et. al., "Near-Fields driving of a optical monopole antenna," J. Opt. A: Pure Appl. Opt., vol. 9, pp. S315-S321, 2007.

[4] B. Hecht, et. al., "Prospects of resonant optical antennas for nano-analysis," Chimia, vol. 60(11), pp. 765-769, 2006.

[5] H. Fischer, and O. J. F. Martin, "Engineering the optical response of plasmonic nanoantennas," Opt. Express, vol. 16(12), pp. 9144-9154, 2008.

[6] R. Kappeler, et. al., "Field computation of optical antennas," J. Comput. Theor. Nanosci., vol. 4(3), pp. 686-691, 2007.

[7] O. Sqalli, I. Utke, P. Hoffmann, and F. M. Weible, "Gold elliptical nanoantennas as probes for near field optical microscopy," J. Appl. Phy., vol. 92(2), pp. 1078-1083, Jul. 2003.

[8] W. Rechberger, et al., "Optical properties of two interacting gold nanoparticles," Opt. Commu., vol. 220, pp. 137-141, 2003.

[9] J. Nelayah, et. al., "Mapping surface plasmons on a single metallic nanoparticle," Nat. Phy., vol. 3, pp. 248-353, May 2007.

[10] V. Myroshnychenko, et. Al, "Modelling the optical response of gold nanoparticles," Chem. Soc. Rev., vol. 37, pp. 1792$1805,2008$.

[11] D. E. Livesay, and K. M. Chen, "Electromagnetic fields induced inside arbitrary shaped biological bodies," IEEE Trans. Micro. Theo. Thec., vol. 22(12), pp. 1273-1280, Dec. 1974.

[12] C. A. Balanis, Antenna Theory: Analysis and Design, 3rd ed., New Jersey: John Wiley, 2005.

[13] J. A. Stratton, Electromagnetic Theory, New York: McGraw-Hill, 1941.

[14] K. Q. da Costa, and V. Dmitriev, "Resonances of modified bowtie nanoparticles with higher field enhancements", to be published in the ITS 2010 - International Telecommunications Symposium.

[15] J. Aizpurua, G. W. Bryant, L. J. Richter, and F. J. G. Abajo, "Optical properties of coupled metallic nanorods for fieldenhanced spectroscopy," Phys. Review B, vol. 71, 2005. 